\title{
Research Paper \\ The Effect of Acupressure at P6 Point on Anxiety and Depression in Hemodialysis Patients
}

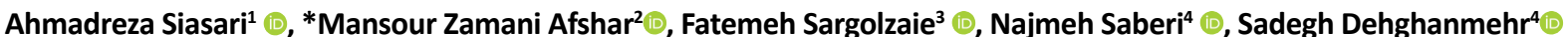

1. Department of Nursing, School of Medicin, Zabol of Medical sciences, Zabol, Iran.

2. Department of Phychology, Payambar Azam Hospital, Zahedan of Medical Sciences, Zahedan, Iran.

3. Department of Nursing, School of Medicin, Hamedan of Medical Sciences, Hamedan, Iran.

4. Department of Nursing, School of Medicin, Zahedan of Medical Sciences, Zahedan, Iran.

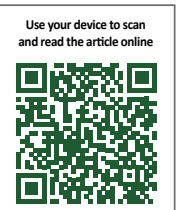

Cttation: Siasari A, Zamani Afsha M, Sargolzaie F, Saberi N, Dehghanmehr S. [The Effect of Acupressure at P6 Point on Anxiety and Depression in Hemodialysis Patients (Persian)]. Complementary Medicine Journal. 2020; 10(2):174-183. https://doi. org/10.32598/cmja.10.2.965.1

https://doi.org/10.32598/cmja.10.2.965.1

Key words: Hemodialysis, Anxiety, Depression, Acupressure, p6 point

\section{A B STRACT}

Objective Anxiety and depression are the most common psychological problems and effective on treatment process in hemodialysis patients. Acupressure is inexpensive, effective, and available method for reducing anxiety and depression. Therefore, this study was conducted to determine the effect of acupressure on anxiety and depression in hemodialysis patients.

Methods This study was a clinical trial, conducted on 40 patient's referral to Imam Khomeini medical center in Zabul at 2017. Patient-based census method selected and randomly divided in two groups (intervention group: 20, control group: 20). For data collection we used demographic, Spielberger Anxiety and Beck Depression Questionnaire. The intervention group received acupressure in p6 point in three days a week for four weeks. Data were collected before and one month after the intervention then data analyzed by SPSS V. 16 with paired t-test and independent statistical test.

Results The Mean $\pm S D$ of depression and anxiety score after intervention were $25.45 \pm 5.67$ and 37.15 \pm 5.13 , respectively. Also the Mean $\pm S D$ of anxiety scores in the experimental and control groups after intervention were $38.2 \pm 4.26$ and $50.6 \pm 7.42$, respectively. Evaluation of depression and anxiety scores after intervention showed a significant difference $(P<0.05)$.

Conclusion The results of this study indicated that acupressure is effective in reducing anxiety and depression in patients. Therefore, it is recommended as a simple, inexpensive, and workable method by the patient himself.

\section{Extended Abstract}

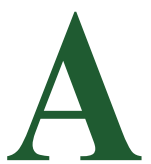

\section{Introduction}

nxiety and depression are among the most important psychological complications in patients undergoing hemodialysis. The prevalence of depression is between 20 to 30 and in some studies up to $88 \%$ and the prevalence of anxiety in hemodialysis patients is between 42.3 to $92.5 \%$ [7, 10-12]. Acupressure is a non-invasive method for boosting the level of the immune system and also psychologically in patients stimulating alpha waves in the brain and causing relaxation and reducing anxiety and depression [9, 19]. Due to the discrepancy in the findings of other studies on the effect

\section{* Corresponding Author:}

Mansour Zamani Afshar, MSc.

Address: Department of Phychology, Payambar Azam Hospital, Zahedan of Medical Sciences, Zahedan, Iran.

Tel: +98 (915) 4375752

E-mail: abcdeh2130@gmail.com 
or non-effect of acupressure on anxiety and depression in patients [20-22] and the limitations of studies in patients undergoing hemodialysis and given that acupressure is a The method was easy, cheap and fast, the aim of this study was to determine the effect of acupressure on anxiety and depression in patients undergoing hemodialysis.

\section{Materials and Methods}

This study is a randomized clinical trial. The sample size required in this study was 40 people. The instruments of this study included demographic information questionnaire, Spielberger anxiety questionnaire, and Beck depression questionnaire. Questionnaires were filled out by research units before the intervention. In the intervention group, acupressure in the form of pressure in the amount of 3 to $4 \mathrm{~kg}$, for eight minutes with the thumb (three minutes at each point and two minutes of rest) in a circular motion and two cycles per second, one hour after the start of dialysis, was performed three days a week for four weeks. After the intervention (after the end of four weeks), the questionnaires were completed again by the patients. For patients in the control group, routine interventions were performed as before. Data were described using descriptive statistics (central indices, dispersion, frequency and percentage) and inferential statistics (independent and paired t-test). To compare the anxiety and depression scores between the two groups, before and after the study, t-test was used and to compare the anxiety and depression of patients in each group, before and after the intervention, paired t-test was used.

\section{Results}

The two groups were homogeneous in terms of demographic information. Evaluation of statistical data before and after the intervention in the intervention and control groups after acupressure showed a statistically significant difference in reducing depression and anxiety based on independent t-test $(\mathrm{P}<0.5)$. Also, comparison of data before and after the intervention in the intervention and control groups based on paired T-test showed a statistically significant difference between anxiety and depression scores before and after the intervention in the intervention group $(\mathrm{P}<0.5)$ and this difference in The control group was not significant (Table $1 \& 2$ ).

\section{Discussion}

The results of the present study showed that acupressure at point p6 have a positive effect on reducing anxiety and depression in patients undergoing hemodialysis. In fact, acupressure is an easy, cheap and simple method and no side effects have been reported for it and it can be easily performed by nurses. It can even be entrusted to patients by teaching it to patients [32].

Various studies have examined the positive effects of acupressure, including the results of a study by Moradi et al., Which evaluated acupressure to reduce anxiety in nulliparous women; Acupressure at $\mathrm{p} 6$ reduces the level of anxiety in patients [33]. The results of the study by Joseph et al., Who evaluated the 66 point in reducing students 'anxiety, also showed the positive effect of acupressure on reduc-

Table 1. Comparison of mean and standard deviation of patients' anxiety scores before and after acupressure

\begin{tabular}{|c|c|c|c|}
\hline \multirow{2}{*}{ Group } & \multicolumn{2}{|c|}{ Mean $\pm S D$} & \multirow[t]{2}{*}{ Paired t-test } \\
\hline & Before & After & \\
\hline Intervention & $47.60 \pm 7.04$ & $38.2 \pm 4.26$ & $P=0.003$ \\
\hline Control & $51.45 \pm 6.08$ & $50.6 \pm 7.42$ & $P=0.02$ \\
\hline Independent t-test & $P=0.1$ & $P=0.04$ & \\
\hline
\end{tabular}
说

Table 2. Comparison of mean and standard deviation of patients' depression score before and after intervention

\begin{tabular}{ccccc}
\hline \multirow{2}{*}{ Group } & \multirow{2}{*}{ Time } & \multicolumn{2}{c}{ Mean \pm SD } & \multirow{2}{*}{ Paired t-test } \\
\cline { 3 - 5 } & & Before & After & \\
\hline Intervention & $31.85 \pm 4.05$ & $28.45 \pm 5.67$ & $\mathrm{P}=0.005$ \\
Control & $33.75 \pm 3.41$ & $37.15 \pm 5.13$ & $\mathrm{P}=0.08$ \\
Independent t-test & $\mathrm{P}=0.07$ & $\mathrm{P}=0.001$ & \\
\hline
\end{tabular}


ing students' anxiety [20] in this regard, HMWE, which evaluated different points of acupressure in reducing depression and anxiety of patients, also showed a decrease in depression and anxiety of patients after the intervention and showed a statistically significant difference [9]. However, in this regard, the results of a study conducted by Mousavi et al. To evaluate acupressure in reducing students' anxiety did not show a statistically significant difference [23].

\section{Conclusion}

The results of the present study and the significant effect of acupressure at point P6 on reducing anxiety and depression in patients undergoing hemodialysis and the high prevalence of these mental disorders in these patients, as well as the ease, simplicity and cheapness of this method, can be recommended to nurses by learning And acupressure at this point, reduce the level of anxiety and depression in these patients. It is also recommended that more research be done in larger volumes and in other parts of acupressure.

\section{Ethical Considerations}

Compliance with ethical guidelines

The present study was registered (Ethics Code zbmu.1.REC.2017.184) and the Clinical Trial Code Number (IRCT20160605028255N2).

Funding

This research did not receive any grant from funding agencies in the public, commercial, or non-profit sectors.

\section{Authors' contributions}

Plan development: Ahmadreza Siasari; Final edition of statistical analysis: Mansour Zamani Afshar; Participation in research: Fatemeh Sargolzaei; Research: Sadegh Dehghan Mehr; Data collection: Najmeh Saberi.

\section{Conflicts of interest}

The authors declared no conflict of interest.

Acknowledgements

The Vice Chancellor for Research of Zabol University of Medical Sciences is thanked for the financial support of this project and also for all the staff and patients of the Hemodialysis Center of Imam Khomeini Hospital in Zabol who helped us in carrying out this research. 


\title{
بررسى تأثير طب فشارى در نقطه P7، بر اضطراب و افسردگى بيماران تحت درمان با همودياليز
}

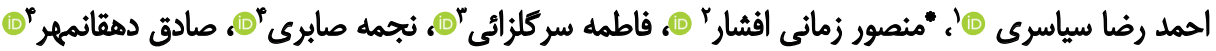

1. أ.روه يرستارى، دانشكده يزشكى، دانشكاه علوم يزشكى زابل، زابل، اير ان.

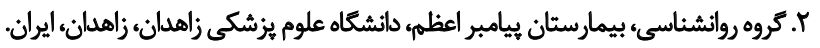

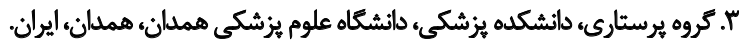

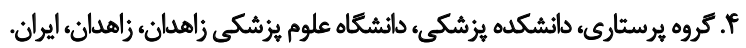

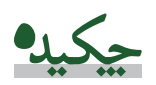

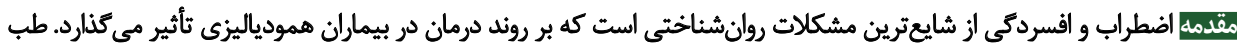

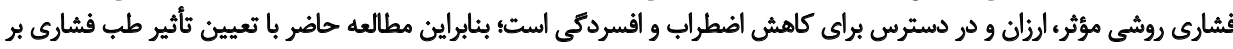

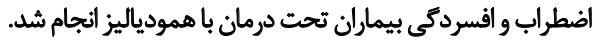

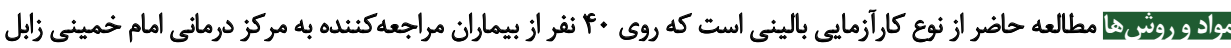

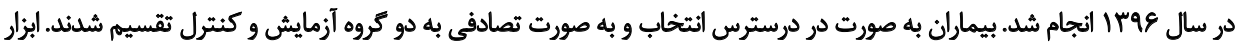

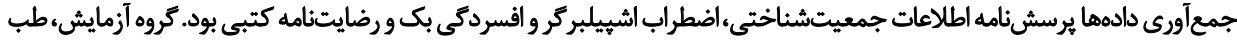

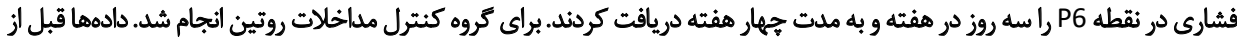

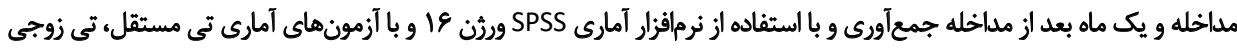
آناليز شدند.

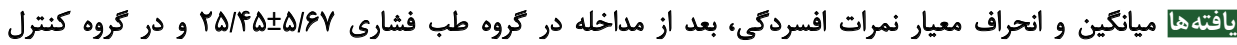

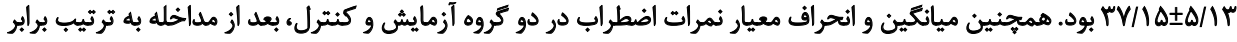

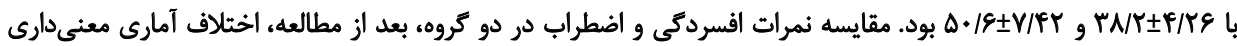

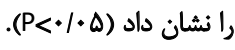

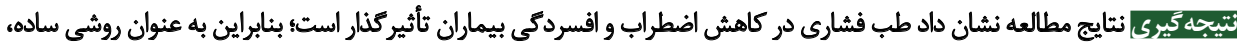

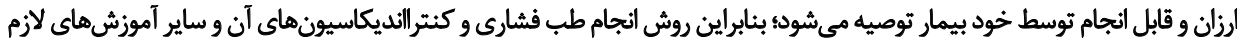

$$
\text { به بيماران توصيه مي أنود. }
$$
\end{abstract}

اطلاعات مقاله:

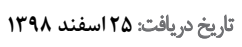

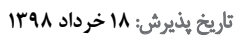

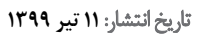

كليدوازوهها:

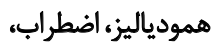

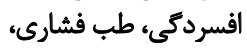

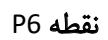

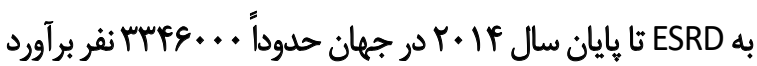

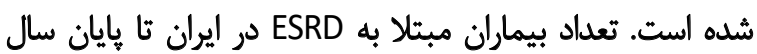

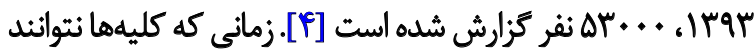

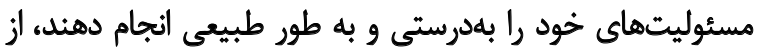

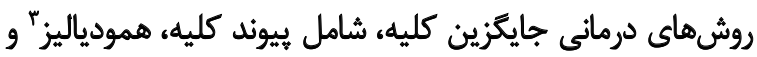

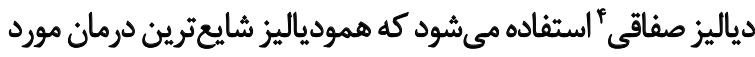

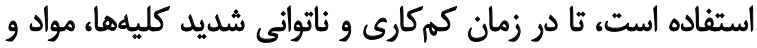

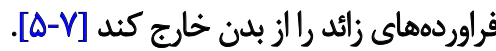

3. Hemodialysis

4. Peritoneal dialysis
مقدمه

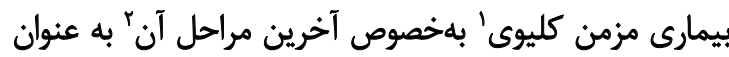

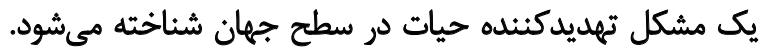

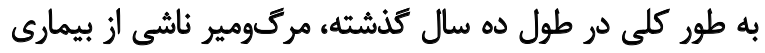

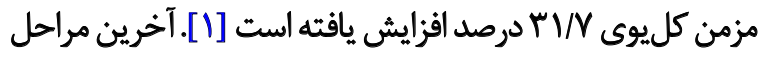

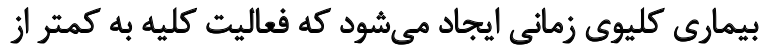

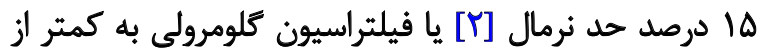

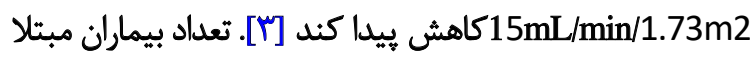

1. Chronic Kidney Disease (CKD)

2. End-Stage Renal Disease (ESRD)

$$
\text { ㄷ... }
$$

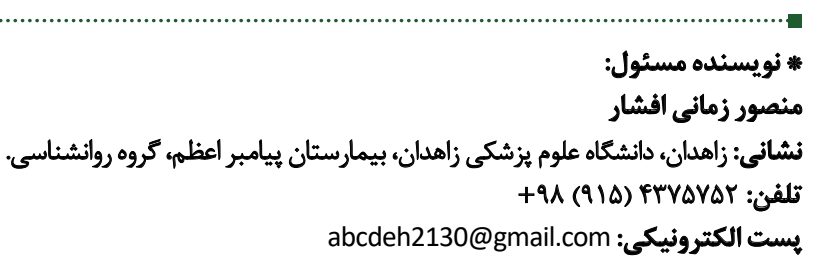


مطالعات مختلفي وجود دارد كه تأثير طب فشارى را بر اضطراب

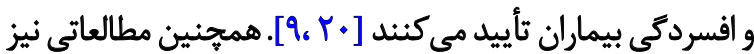

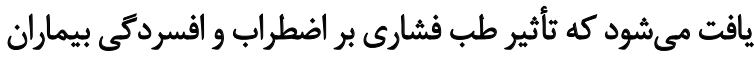

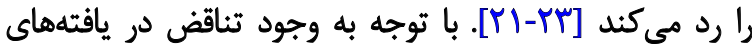

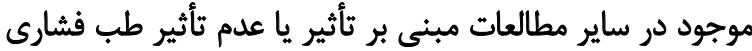

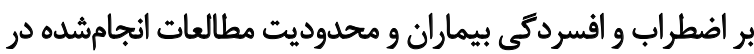

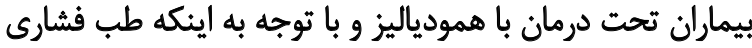

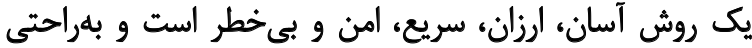

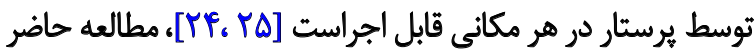

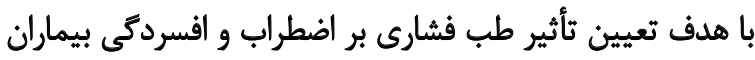
تحت درمان با همودياليز انجام شد.

\section{مواد و روشها}

اين مطالعه، يك مطالعه كارآزمايى بالينى تصادفى است. حجم إنما

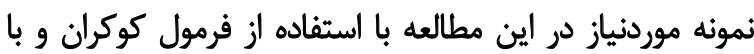
احتساب

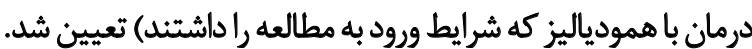

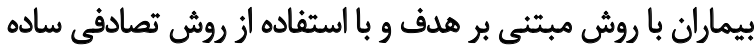

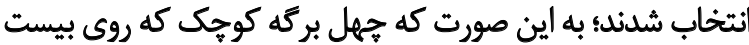

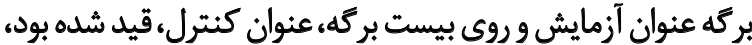

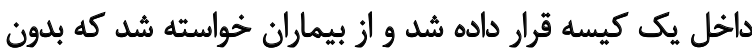

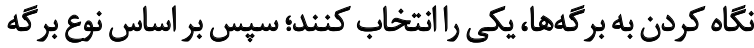

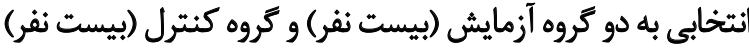

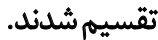

معيارهاى ورود به اين مطالعه عبارت بودند از: داشتن حداقل هجده سال سن، حداقل شش ماه سابقه همودياليز، دائ داشتن يرائ برونده

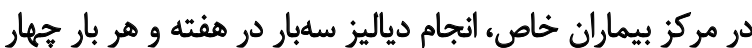

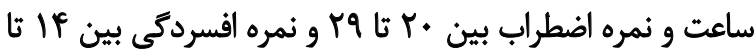

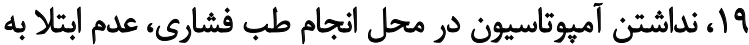

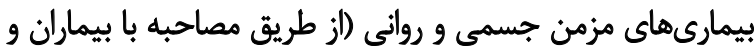

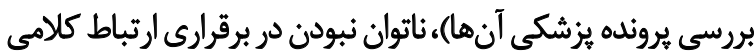

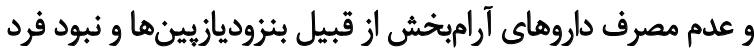

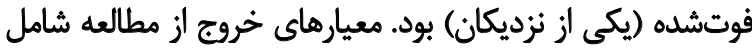

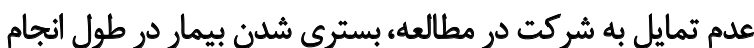
مداخله، مسافرت و فوت بيمار بودند.

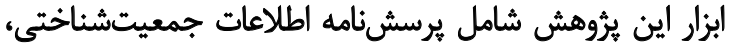

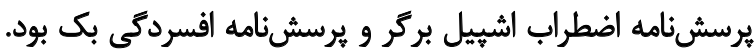

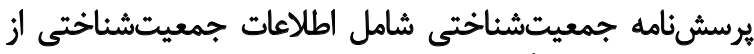
قبيل سن، جنس، تأهل وغيره بودن

جهت اندازهيرى سطح اضطراب بيماران از برسشنامه اشيّيل

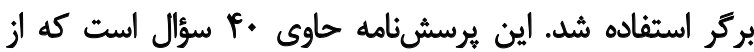

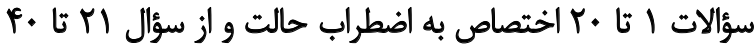

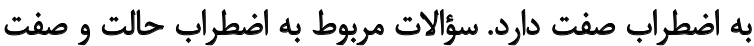

بيماران تحت درمان با همودياليز داراي محدوديتها و مشكلات

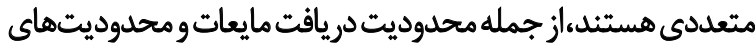

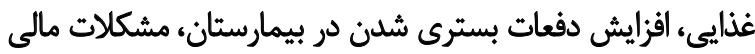

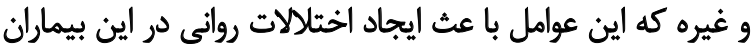

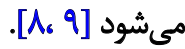

اضطراب و افسردكى از جمله مههمترين عوارض روائى ايجادشده

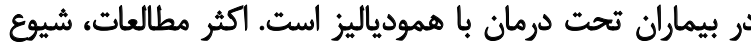

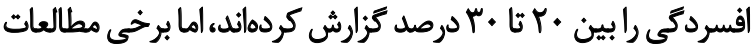

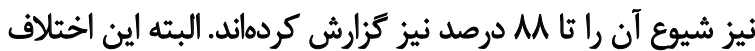

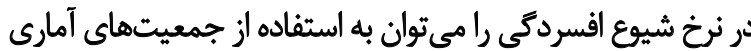

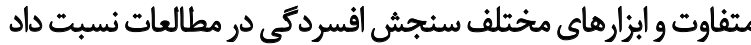

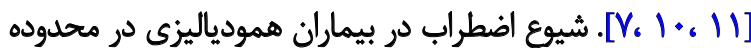

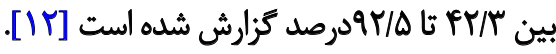
از آنجايى كه اضطراب و افسردمى مى توانند عوارض جبران نايذيرى

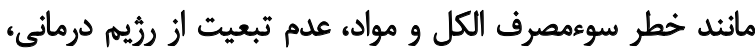

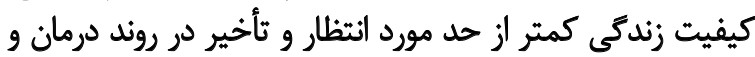

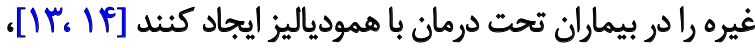
بنابراين براى جلوكيرى از اين عوارض بايد تحت دان درمان قرار تيرند. درمان اضطراب و افسردگى به دو صورت دارويى و غيردارويى انجام

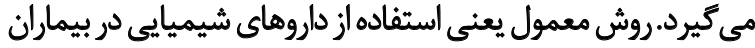

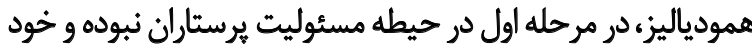

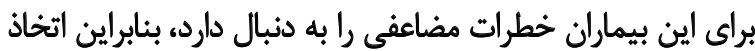

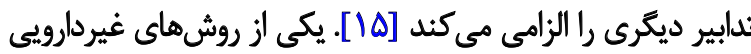

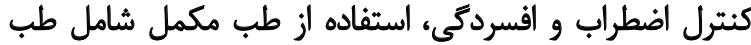

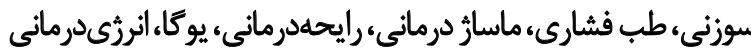

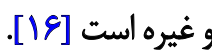

طب فشارى يكى از زيرشاخههاى اصلى طب مكمل و از

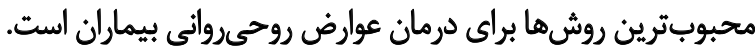

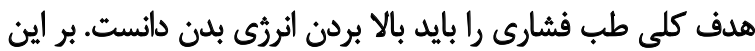

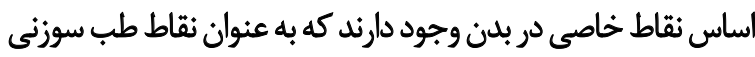

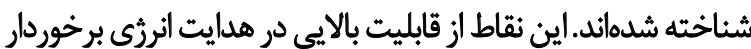

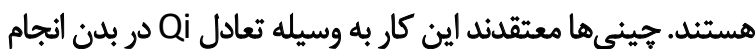

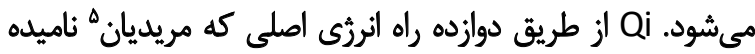

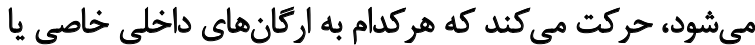

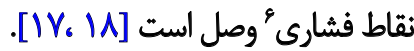
طب فشارى يك روش غيرتهاجمى براى ارتقاى سطح سيستم

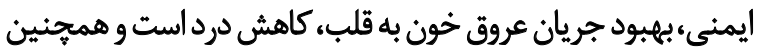

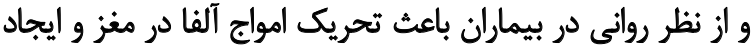

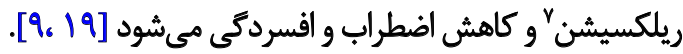

5. Meridian

6. Acupoint

7. Relaxation 


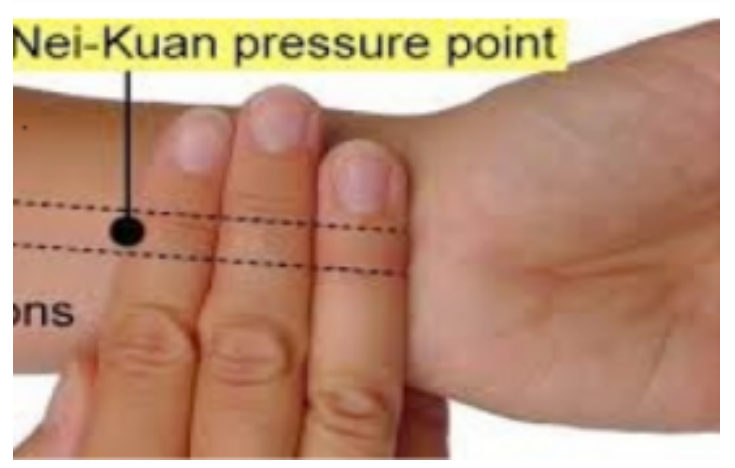

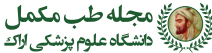

تصوير ا. محل انجام طب فشارى در نقطه p6

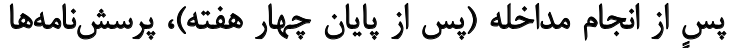

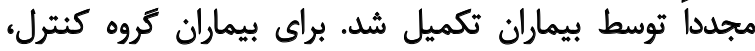

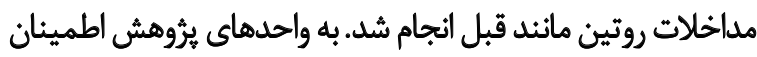

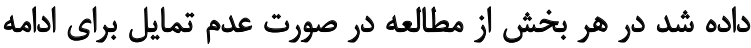
مطالعه، مى توانند ازئ مطالعه خارج شوند. دادهها يّ از جمع آورى وارد نرمافزار SPSS شدند. توصيف دادهها

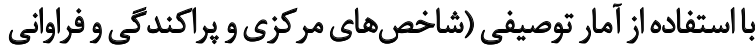

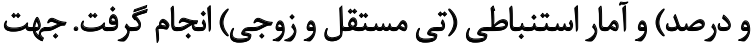

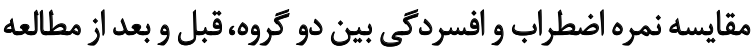

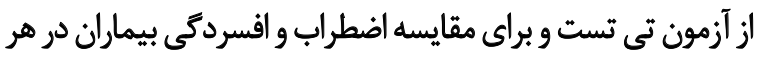

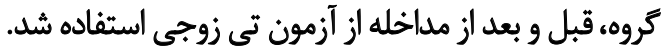

بافتهها

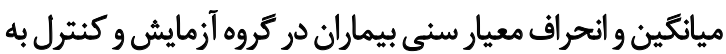

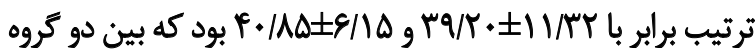

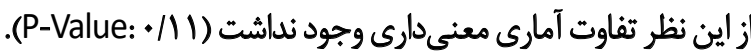

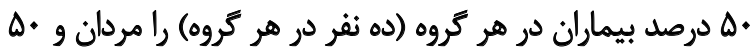

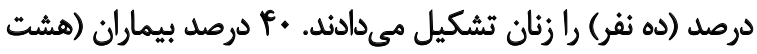

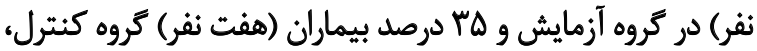

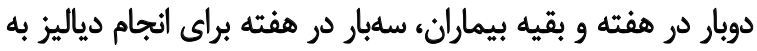

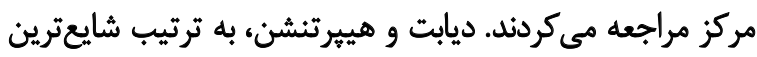

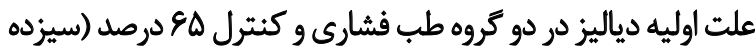

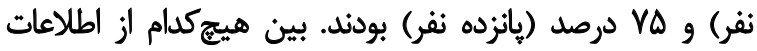

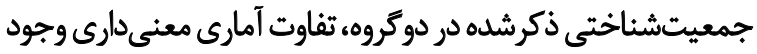

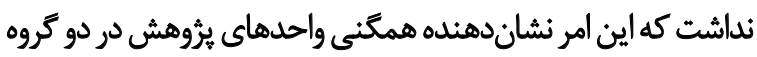
است (جدول شماره ()).

ارزيابي دادههاي آمارى قبل و بعد از مداخله در دو كروه آزمايش و

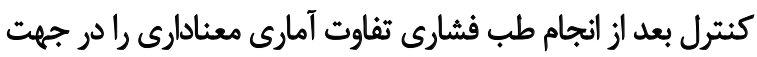

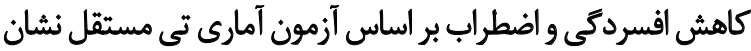

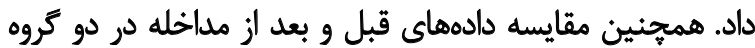

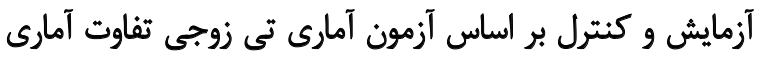

در طيف ليكرت جهاركزينه اى نمرهكذارى مى دوند. هر فرد نيز

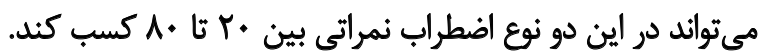

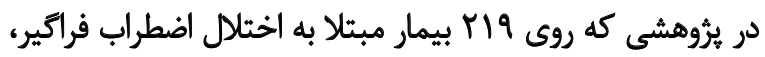

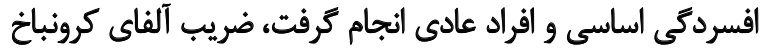

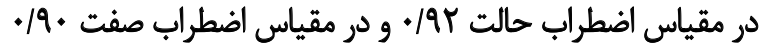

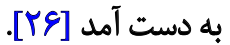

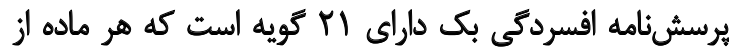

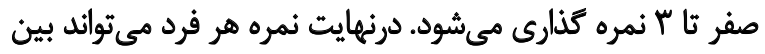

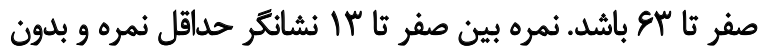

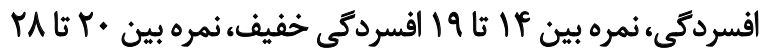

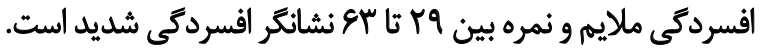

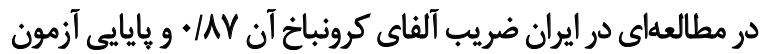

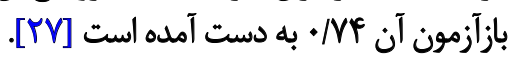

انجام طب فشارى در اين مطالعه در كروه آزمايش روى نقطه

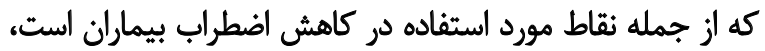

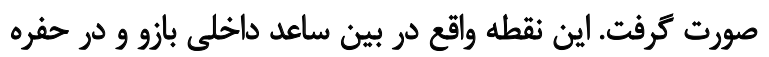

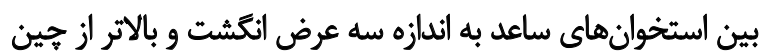

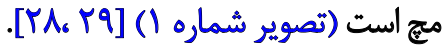

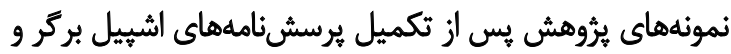

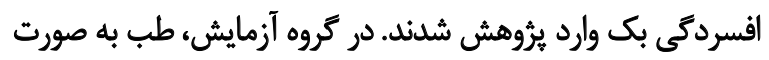

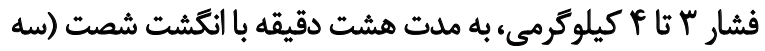

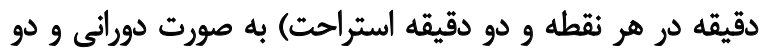

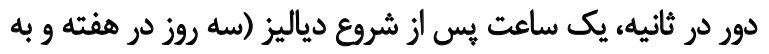

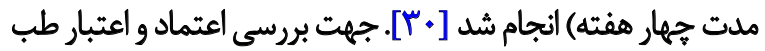

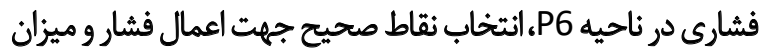

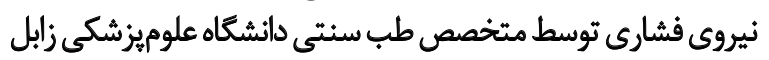

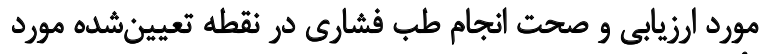

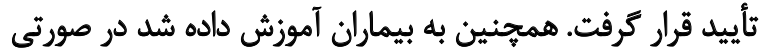

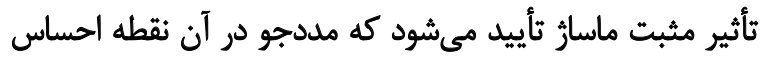

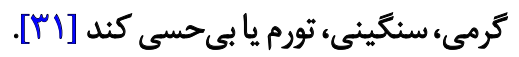


جدول ا. مشخصات جمعيتشناختى بيماران در كروه آزمايش و كثترل

\begin{tabular}{|c|c|c|c|c|}
\hline \multirow{2}{*}{$\mathbf{P}$} & \multicolumn{2}{|c|}{ ميانيين]|نحراف معيار/ تعداد (درصد) } & \multirow{2}{*}{\multicolumn{2}{|c|}{ متغير }} \\
\hline & كئروه كنترل & كروه آزمايش & & \\
\hline .111 & $r+11 \Delta \pm \pm / 10$ & $r q / r \cdot \pm 11 / \pi r$ & & \\
\hline 1 & $\begin{array}{l}1 \cdot(\Delta \cdot) \\
1 \cdot(\Delta \cdot)\end{array}$ & $\begin{array}{l}1 \cdot\left(\Delta^{*}\right) \\
1 \cdot\left(\Delta^{*}\right)\end{array}$ & $\begin{array}{l}\text { هرد } \\
\text { نز }\end{array}$ & جنسيت \\
\hline .11 & $\begin{array}{l}V(r \Delta) \\
\operatorname{Ir}(\varepsilon \Delta)\end{array}$ & $\begin{array}{l}\Lambda\left(f^{*}\right) \\
M(\varepsilon \cdot)\end{array}$ & دوبار & تعلاد دفعات دياليز در \\
\hline .1 .9 & $\begin{array}{l}\varepsilon(r \cdot) \\
\Lambda(r \cdot) \\
\Delta(r \Delta) \\
I(\Delta)\end{array}$ & $\begin{array}{l}V(r \Delta) \\
V(r \Delta) \\
\Delta(r \Delta) \\
I(\Delta)\end{array}$ & بيرفارى بيمارى كليوى & علت اوليه دياليز \\
\hline
\end{tabular}

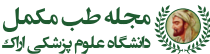

جدول r. مقايسه ميانكين و انحراف معيار نمره اضطراب بيماران قبل و بعد از مداخله طب فشارى در كروه آزمايش و كنترل

\begin{tabular}{|c|c|c|c|}
\hline \multirow{2}{*}{ أزمون تى زوج } & \multicolumn{2}{|c|}{ ميانكين||نحراف معيار } & \multirow[t]{2}{*}{ زمان } \\
\hline & يس از مداخله & قبل از مداخله & \\
\hline$P=+1+\cdot r$ & TNTIF/TE & $F V / \varepsilon+ \pm V / \cdot F$ & آزمايش \\
\hline \multirow[t]{2}{*}{$\mathrm{P}=\cdot / \pi$} & $\Delta \cdot / 8 \pm V / e r$ & $\Delta \backslash / \mathscr{f} \Delta \pm \varepsilon / \cdot A$ & كنترل \\
\hline & $\mathrm{P}=* / * f$ & $P=+11$ & أزمون تى مستقل \\
\hline
\end{tabular}

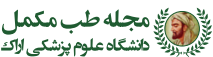

همودياليز مىشود.درواقع طب فشارى يك روش آسان، ارزان و ساده

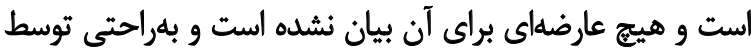

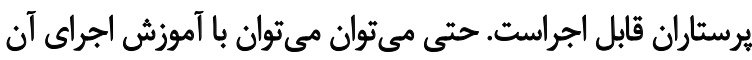
به بيماران انجام آن را به خود بيماران سيرد [بست]

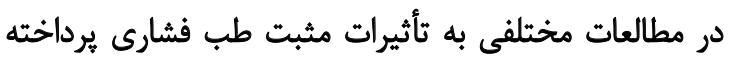

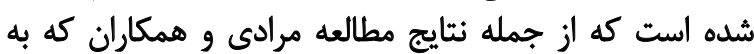
الدزيابى انجام طب فشارى در كاهش اضطراب زناب مران نخستزال
معنادارى را بين نمرات اضطراب و افسردكى قبل و بعد از مداخله در

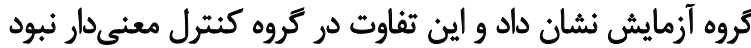

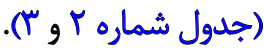

بحث

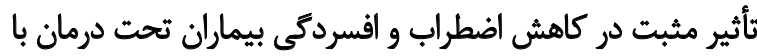

جدول r. مقايسه ميانكين و انحراف معيار نمره افسردكى بيماران قبل و بعد از مداخله در كروه آزمايش و كئترل

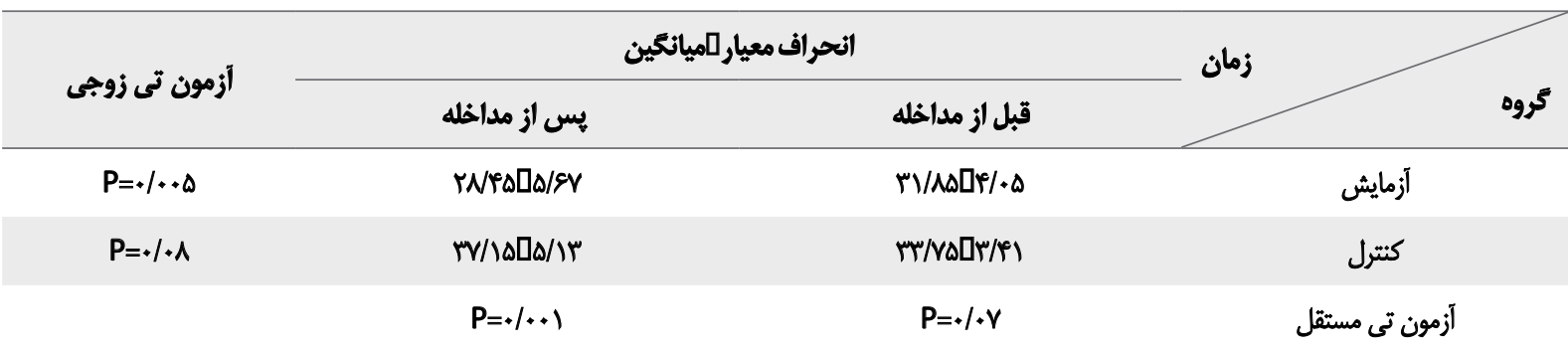

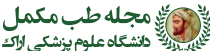




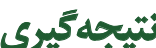

با توجه به نتايج ثرؤش حاض حاضر و تأثير معنى دار طب فشارى

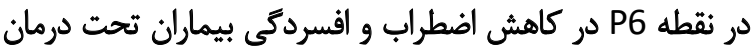

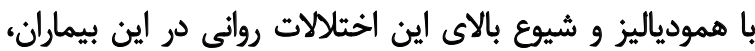

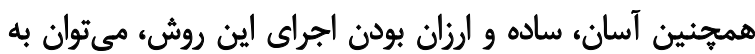

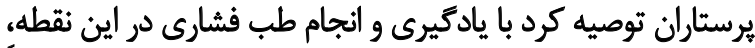

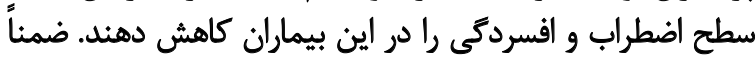

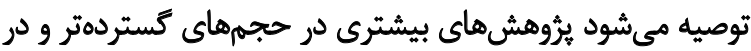
ساير نقاط طب فشارى انجام شود.

مالاحظات اخلاقي

\section{بيروى از أصول اخلاق بثروهش}

اين مقاله بركرفته از طرح دانشجويى مصوب دانشكاه

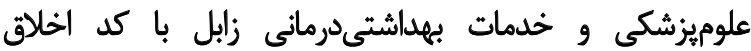

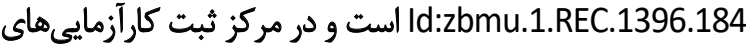

بالينى نيز با كد IRCT20160605028255N2 ثبت شده است.

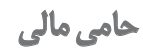

معاونت يرؤهشى دانشكاه علوميزشكى و خدمات بهداشتى زابل به حامى مالى اين يُّوهش بوده است.

$$
\text { مشاركت نويسئدكان }
$$

تدوين اوليه طرح: محمدرضا سياسرى؛ ويرايش نهايي مقاله وآناليز

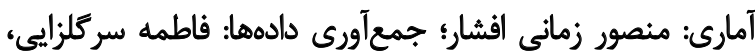
صادق دهقانمهر و نجمه صابرى.

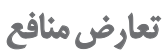

بنابر اظهار نويسئدكان اين مقاله تعارض منافع ندارد.

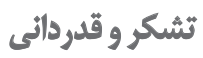

از از اين طرح و همجينين از تمامى برسنل و بيماران مركز

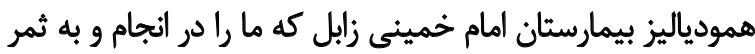
رساندن اين يثوهش يارى كردند، صميمانه تقدير و تشكر مى كنيهم.
يرداخته بودهاند، نشان داد انجام طب فشارى در نقطه P6 باعث

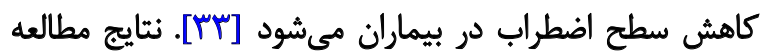

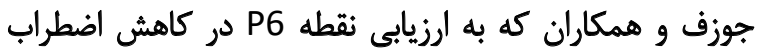

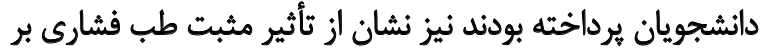

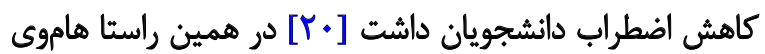

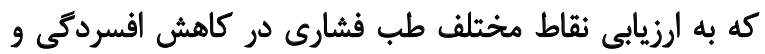

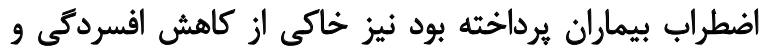

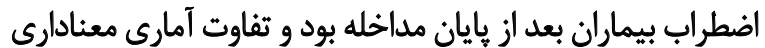

را نشان داد [9].

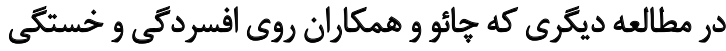

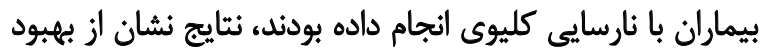

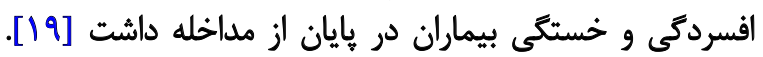

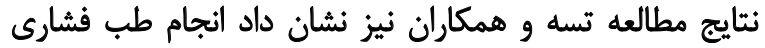

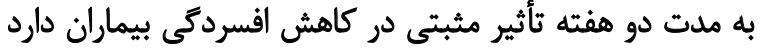

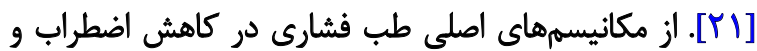

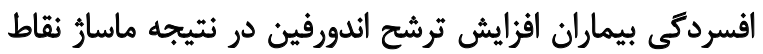

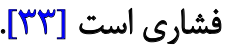

تئورى ديكرى در علوميزشكى نيز معتقد است كه طب فشارى

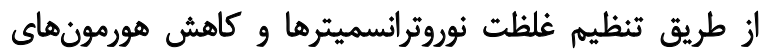

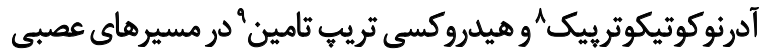

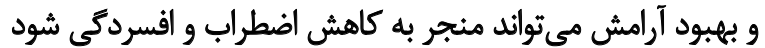

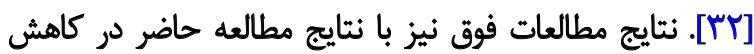
افسردكى و اضطراب بيماران در يك راستاست.

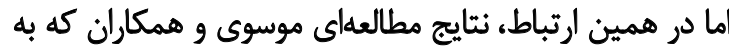

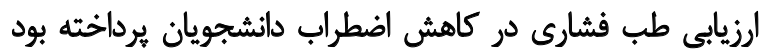

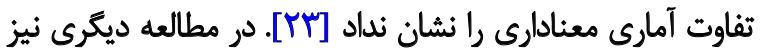

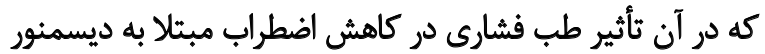

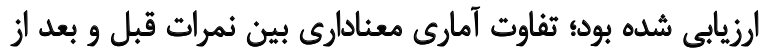

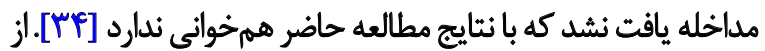

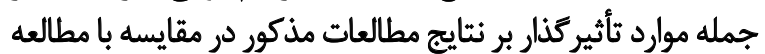

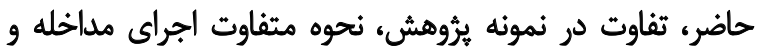
مدتزمان و تعداد جلسات آن است. با توجه به نتايج اين مطالعه و ساير مطالعات انجامشده در اين

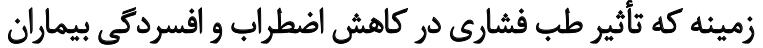

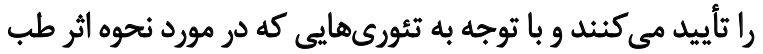

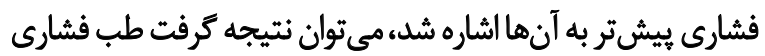

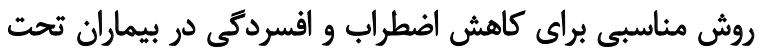

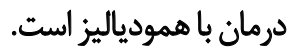




\section{References}

[1] Rehman IUP, Wu DBC, Ahmed R, Khan NA, Rahman AU, Munib S, et al. A randomized controlled trial for effectiveness of zolpidem versus acupressure on sleep in hemodialysis patients having chronic kidney disease-associated pruritus. Medicine. 2018; 97(31):e10764. [DOI:10.1097/MD.0000000000010764] [PMID] [PMCID]

[2] Jones DJW, Butler LT, Harris JP, Vaux EC. Latent learning in End Stage Renal Disease (ESRD). Physiology \& Behavior. 2015; 142:42-7. [DOI:10.1016/j.physbeh.2015.01.033] [PMID]

[3] Palmer SC, Natale P, Ruospo M, Saglimbene VM, Rabindranath KS, Craig JC, et al. Antidepressants for treating depression in adults with end-stage kidney disease treated with dialysis. The Cochrane Database of Systematic Reviews. 2016; (5):CD004541. [DOI:10.1002/14651858. CD004541.pub3] [PMID]

[4] Mirbagher-Ajorpaz N, Aghajani M, Morsaee F, Zabolian Zh. [The relationship between hope and depression-anxiety in patients undergoing hemodialysis (Persian)]. Journal of Health and Care. 2016; 18(1):55-62. http://hcjournal.arums.ac.ir/article-1-437-en.html

[5] Rahdar Z, Jahantigh Haghighi M, Mansouri A, Siasary AR, Alahyari J, Jahantigh $F$. Probing the relationship between treatment regimen compliance and the quality of life in hemodialysis patients: A descriptiveanalytic study. Medical - Surgical Nursing Journal. 2019; 8(2):e95599. [DOI:10.5812/msnj.95599]

[6] Bouya S, Allahyari J, Hesaraki M, Koochakzai M, Maleknejad A. The importance of health literacy in patients with chronic kidney disease: A literature review. La Prensa Médica Argentina. 2018; 104(6):1000321. https://www.researchgate.net/publication/330637225

[7] Delshad Noughabi A, Ghorbany Moghadam I, Khavasi M, Raznahan R, Abbasi Mendi A, Mohsenikhah M. [Evaluation of depression, anxiety and stress in hemodialysis patients in Gonabad City 2017 (Persian)]. The Horizon of Medical Sciences. 2019; 25(2):134-8. http://hms.gmu.ac.ir/ article-1-3064-en.htm

[8] Ahmad MM, Al Nazly EK. Hemodialysis: Stressors and coping strategies. Psychology, Health \& Medicine. 2015; 20(4):477-87. [DOI:10.1080/1354 8506.2014.952239] [PMID]

[9] Hmwe NTT, Subramanian P, Tan LP, Chong WK. The effects of acupressure on depression, anxiety and stress in patients with hemodialysis: A randomized controlled trial. International Journal of Nursing Studies. 2015; 52(2):509-18. [DOI:10.1016/j.ijnurstu.2014.11.002] [PMID]

[10] Najafi A, Keihani S, Bagheri N, Ghanbari Jolfaei A, Mazaheri Meybodi A. Association between anxiety and depression with dialysis adequacy in patients on maintenance hemodialysis. Iranian Journal of Psychiatry and Behavioral Sciences. 2016; 10(2):e4962. [DOI:10.17795/ijpbs-4962] [PMID] [PMCID]

[11] Shafipour V, Alhani F, Kazemnejad A. A survey of the quality of life in patients undergoing hemodialysis and its association with depression, anxiety and stress. Journal of Nursing and Midwifery Sciences. 2015; 2(2):29-35. [DOI:10.4103/2345-5756.231432]

[12] Dehghanmehr S, Mansouri A, Faghihi H, Piri F. The effect of acupressure on the anxiety of patients undergoing hemodialysis -a review. Journal of Pharmaceutical Sciences and Research. 2017; 9(12):2580-4. https://www.jpsr.pharmainfo.in/Documents/Volumes/vol9lssue12/ jpsr09121758.pdf

[13] Masinaienejad N, Abdollahi Mohammad A, Jahantigh F, Zamani Afshar M, Allahyari J. The impact of self-care education based on Orem's model on self-care behaviors of patients with $\beta$-Thalassemia major: A clinical trial. Medical - Surgical Nursing Journal. 2019; 8(3):e96823. [DOI:10.5812/msnj.96823]
[14] Cohen SD, Cukor D, Kimmel PL. Anxiety in patients treated with hemodialysis. Clinical Journal of the American Society of Nephrology. 2016; 11(12):2250-5. [DOI:10.2215/CJN.02590316] [PMID] [PMCID]

[15] Dehghanmehr S, Sheikh Gh, Siyasari AR, Sheikh A, Nooraien S, Salarzaei $M$. The impact of foot reflexology massage on anxiety caused by blood transfusion in children with thalassemia. International Journal of Pharmaceutical Sciences and Research. 2018; 9(5):2053-7. https://ijpsr com/bft-article/the-impact-of-foot-reflexology-massage-on-anxietycaused-by-blood-transfusion-in-children-with-thalassemia/

[16] Tayebi A, Kasra Dehkordi AR, Ebadi A, Sahraei H, Einollahi B. [The effect of aromatherapy with lavender essential oil on depression, anxiety and stress in hemodialysis patients: A clinical trial (Persian)]. Evidence Based Care Journal. 2015; 5(2):65-74. [DOI:10.22038/EBCJ.2015.4549]

[17] Dehghanmehr S, Sargazi GH, Biabani A, Nooraein S, Allahyari J. Comparing the effect of acupressure and foot reflexology on anxiety and depression in hemodialysis patients: A clinical trial. Medical - Surgical Nursing Journal. 2020; 8(4):e100386. [DOI:10.5812/msnj.100386]

[18] Kao CL, Chen CH, Lin WY, Chiao YC, Hsieh CL. Effect of auricular acupressure on Peri- and early postmenopausal women with anxiety: A double-blinded, randomized, and controlled pilot study. EvidenceBased Complementary and Alternative Medicine. 2012; 2012:567639. [DOI:10.1155/2012/567639] [PMID] [PMCID]

[19] Cho YC, Tsay SL. The effect of acupressure with massage on fatigue and depression in patients with end-stage renal disease. Journal of Nursing Research. 2004; 12(1):51-4. [DOI:10.1097/01. JNR.0000387488.63438.9a] [PMID]

[20] Joseph VM. Effect of self acupressure on anxiety among the nursing students undertaking their university examination in a selected nursing college, Tamil Nadu, India. Indian Journal of Applied Research. 2015; 5(9):436-7. https://www.worldwidejournals.com/indian-journal-ofapplied-research-(IJAR)/fileview/September_2015_1492583367 201. pdf

[21] Tse M, Au J. The effects of acupressure in older adults with chronic knee pain: Depression, pain, activities of daily living and mobility. Journal of Pain Management. 2010; 3(4):339-410. http://ira.lib.polyu.edu. hk/handle/10397/19615

[22] Moradi M, Azizi H, Saffari SE, Koushan M. [The effect of acupressure on anxiety in patients with hemodialysis (Persian)]. Journal of Sabzevar University of Medical Sciences. 2015; 22(5):765-72. http://jsums.medsab. ac.ir/article_754.html

[23] Moosavi S, Mirzaei M, Reza Soltani P. [The effects of acupressure on anxiety nursing, midwifery and operating room students (Persian)]. Journal of Guilan University of Medical Sciences. 2009; 18(71):82-9. http://journal.gums.ac.ir/article-1-242-fa.html

[24] Khachian A, Saatchi K, Aghaamoo Sh, Haghani H, Tourdeh M. [Comparison of the effects of acupressure and touch on the headache caused by spinal anesthesia after cesarean section (Persian)]. The Iranian Journal of Obstetrics, Gynecology and Infertility. 2016; 18(184):9-19. [DOI:10.22038/IJOGI.2017.6728]

[25] Mojalli M, Abbasi P, Kianmehr M, Zamani S. [Effect of acupressure on fecal impaction in hemodialysis patients (Persian)]. Journa of Mazandaran University of Medical Sciences. 2016; 26(136):18-25. http://jmums.mazums.ac.ir/article-1-7485-en.html

[26] Wagner J. CE: Incorporating acupressure into nursing practice. The American Journal of Nursing. 2015; 115(12):40-5. [DOI:10.1097/01. NAJ.0000475290.20362.77]

[27] Ghassemzadeh H, Mojtabai R, Karamghadiri N, Ebrahimkhani N. Psychometric properties of a Persian-language version of the Beck depres- 
sion inventory - second edition: BDI-II-Persian. Depression \& Anxiety. 2005; 21(4):185-92. [DOI:10.1002/da.20070] [PMID]

[28] Rajai N, Choopani N, Pishgooie SAH, Sharififar ST. [The effect of P6 acupressure point on physiological indices in coronary angiography candidate (Persian)]. Complementary Medicine Journal. 2016; 5(4):1290-302. http://cmja.arakmu.ac.ir/article-1-356-en.html

[29] Shahdadi H, Shirani N, Mansouri A. [The effect of acupressure on anxiety and quality of life in patients with type II diabetes (Persian)]. Journal of Diabetes Nursing. 2017; 5(4):263-72. http://jdn.zbmu.ac.ir/article1-285-en.html

[30] Arab Z, Shariati AR, Bahrami HR, Asayesh H, Vakili MA. [The effect of acupressure on quality of sleep in hemodialysis patients (Persian)]. Nursing and Midwifery Journal. 2012; 10(2):237-45. http://unmf.umsu. ac.ir/article-1-828-en.html

[31] Shahdadi H, Allah-Yari J, Mansouri A. [Effect of acupressure on sleep quality in patients with diabetes mellitus (Persian)]. Journal of Diabetes Nursing. 2017; 5(2):78-85. http://jdn.zbmu.ac.ir/article-1-250-en.html

[32] 32. Beikmoradi A, Najafi F, Roshanaei Gh, Pour Esmaeil Z, Khatibian $\mathrm{M}$, Ahmadi AR. Acupressure and anxiety in cancer patients. Iranian Red Crescent Medical Journal. 2015; 17(3):e25919. [DOI:10.5812/ ircmj.25919] [PMID] [PMCID]

[33] Moradi Z, Akbarzadeh M, Moradi P, Toosi M, Hadianfard MJ. The effect of acupressure at GB-21 and SP-6 acupoints on anxiety level and maternal-fetal attachment in primiparous women: A randomized controlled clinical trial. Nursing and Midwifery Studies. 2014; 3(3):e19948. [PMID] [PMCID]

[34] Kafaei Atrian M, Sameie A, Sarvieh M, Mirbagher Ajorpaz N, Asghari Jafarabadi M. Comparison of the effect of acupressure at spleen 6 and liver 3 points on the anxiety level of patients with primary dysmenorrhea- Blind randomized controlled clinical trial. Journal of Kermanshah University of Medical Science. 2014; 18(5):e74094. [DOI:10.22110/ jkums.v18i5.1732] 